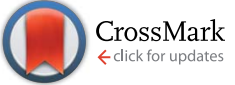

Cite this: RSC Adv., 2017, 7, 17236

Received 12th January 2017

Accepted 24th February 2017

DOI: $10.1039 / c 7 r a 00494 j$

rsc.li/rsc-advances

\section{A study on the identification of habitats and determination of sulfur dioxide residue of Radix Astragali by UV-vis-SWNIR diffuse reflectance spectroscopy}

\begin{abstract}
Xiaoli Jing, ${ }^{a}$ Jie Tang, ${ }^{b}$ Yang Liu, ${ }^{a}$ Xiaomei Lu ${ }^{b}$ and Bing Liang ${ }^{\star b}$
The feasibility of identifying habitat of sulfur fumigated Radix Astragali and of determining its sulfur dioxide residue using UV-vis and short wave near-infrared diffuse reflectance spectra coupled with chemometrics was first investigated. The results show that by using UV-vis-SWNIR DRS combined with PCA, the habitat of Radix Astragali is identified definitely, whether it is sulfur-fumigated, or not sulfur-fumigated; after waveband selection, SG smoothing and excluding outliers, PLS modelling results were improved with a $R^{2}$ of $0.9238,0.9104$ and 0.9322 for calibration set, cross validation set and prediction set, respectively. A convenient and rapid method for identification of the habitat of sulfur fumigated Chinese herbal medicines and determination of sulfur dioxide residue by UV-vis-SWIR DRS was proposed.
\end{abstract}

\section{Introduction}

Identification of herbal medicines is an important part of their quality control. Traditional identification of herbal medicines is based on the difference in source, character identification, physicochemical identification and microscopic identification. ${ }^{1}$ But these methods depend on experience to some extent and may not fully reflect the medicinal quality. ${ }^{2}$ Afterwards, thinlayer chromatography, ${ }^{3-5}$ HPLC $^{6,7}$ and fingerprint technology, ${ }^{8-11}$ based on the extraction of related indexes, were developed and widely used for the identification and quality evaluation of herbal medicines. However, they are complicated, laborious, time-consuming and solvent-consuming due to requiring chemical sample-pretreatments such as extraction, separation, and concentration. ${ }^{2}$ Therefore, in recent years, rapid and nondestructive methods without chemical pretreatment have gained fast development and wide acceptance. Infrared spectroscopy, ${ }^{\mathbf{1 2 - 1 4}}$ Fourier transform infrared (FTIR) spectra, ${ }^{15}$ near infrared reflectance spectroscopy (NIR), ${ }^{\mathbf{1 4 , 1 6 - 1 9}}$ FT-Raman spectroscopy ${ }^{14}$ and $\mathrm{THz}^{-T_{D S}}{ }^{\mathbf{2 , 2 0 , 2 1}}$ methods have been reported for this objective. However, they have some disadvantages such as using sophisticated and expensive instruments. Additionally, they do not include UV-vis spectral region which contains a wealth of information regarding electron-energy level transition of molecules. THz-TDS also has other shortcomings: a lot of Chinese medicines have no obvious characteristic absorption

${ }^{a}$ College of Medical Technology, Chengdu University of Traditional Chinese Medicine, Chengdu 611137, China

${ }^{b}$ School of Chemical Engineering, Sichuan University, Chengdu 610065, P. R. China. E-mail: lbyy0019@sina.com peak in the $\mathrm{THz}$ frequency region, ${ }^{\mathbf{2 0 , 2 2}}$ the strong interference from water absorption makes it need to set the instrumental system in an airtight enclosure with nitrogen gas infusion to keep the relative humidity as less than $8 \%$ even $1 \%$, to overcome the effects of water absorption., 2,20,23

On the other hand, sulfur fumigation was a traditional processing way used widely for some kinds of Chinese medicinal materials (CMM), which can make CMM mothproof and mildewproof, have beautiful appearance and bright color, ${ }^{24,25}$ and is helpful for storage. However, sulfur fumigation may have a great impact on the chemical compositions and pharmacological effects of CMM, ${ }^{24-30}$ make more residue of $\mathrm{SO}_{2}{ }^{30,31}$ and more residues of toxic and harmful substances such as lead, arsenic, mercury and chromium..$^{24,32,33}$

Therefore, control of $\mathrm{SO}_{2}$ residue in CMM is necessary and it has been becoming more and more strictly. China has stipulated that residue amount of sulfur dioxide in herbal medicines should not exceed $150 \mathrm{mg} \mathrm{kg}^{-1}$, sulfur dioxide residue amount in 10 kinds of herbs and slices such as Rhizoma Dioscoreae, Radix Achyranthis Bidentatae, Kudzu root, Radix Asparagi, Rhizoma Gastrodiae, Radix Trichosanthis, Rhizoma Bletillae, Radix Paeoniae Alba, Rhizoma Atractylodis Macrocephalae and Radix Codonopsis should not exceed $400 \mathrm{mg} \mathrm{kg}^{-1} \cdot{ }^{34}$

Chinese official determination methods of $\mathrm{SO}_{2}$ residue in herbal medicines include acid-base titration, gas chromatography and ion chromatography. The first and third need a pretreatment of steam distillation, they are complicated in operations and time-consuming. Therefore, effective and rapid determination of sulfur dioxide residue in herbal medicines becomes a key of quality supervision. It is expected to establish fast and efficient methods. FTIR spectroscopy has been 
reported to be used to identify sun-dried and sulfur-fumigated Radix Paeoniae Alba, ${ }^{35}$ Radix Angelicae Sinensis, ${ }^{36}$ but they needed extraction and didn't determine $\mathrm{SO}_{2}$ residue.

UV-vis-shortwave near infrared diffuse reflectance spectroscopy (UV-vis-SWNIR DRS) has advantages containing the information regarding electron-energy level transition as well as vibrational motion of molecules, being simple, fast and non-destructive. We have reported its applications on qualitative and simultaneous quantitative analysis of cimetidine polymorphs, ${ }^{37}$ qualitative analysis of chiral alanine, ${ }^{38}$ simultaneous determination of amiloride and hydrochlorothiazide in a compound tablet, ${ }^{39}$ qualitative and quantitative analysis of chiral alanine, ${ }^{40}$ rapid and simultaneous determination of soil properties ${ }^{41}$ in which the importance of UV-vis spectral region for analysis has proven. But its use for the identification of habitats of herbal medicines and determination of $\mathrm{SO}_{2}$ residue in herbal medicines has not been reported.

Radix Astragali, a kind of Chinese traditional medicine mainly distributed in North China, has following clinical efficacies: tonifying qi and lifting yang, consolidating exterior for arresting sweating, inducing diuresis for removing edema, creating body fluid and nourishing blood, dissipating stagnation and promoting arthromyodynia, expelling toxin and eliminating pus, restraining ulceration and growing skin. ${ }^{34}$ It can enhance the immune function of patients, viral interferon induction ability, hypoxia tolerance of the patients' body, promote metabolism and the antihypertensive and liver-protecting function. It is widely used in clinical treatment of many diseases. Radix Astragalus is prone to pests during storage.

Therefore, the objective of this study is to establish a simple, fast and non-destructive method to identify habitats of herbal medicines and to determine their $\mathrm{SO}_{2}$ residues, by using UV-visSWNIR DRS combined with chemometrics, and Radix Astragali was used as a research object.

\section{Experimental}

\subsection{Sample and sulfur fumigation}

Radix Astragali from three provinces Sichuan, Shanxi and Gansu were purchased from Wu Kuaishi market of Chinese herbal medicines in Chengdu, Sichuan province. In appearance, Radix Astragali from Sichuan is partial white, Radix Astragali from Gansu and Shanxi are yellowish, as shown in Fig. 1.

About $150 \mathrm{~g}$ of Radix Astragali of each habitat was weighed, wetted by spraying $40 \mathrm{~mL}$ of water and kept in a plastic bag for

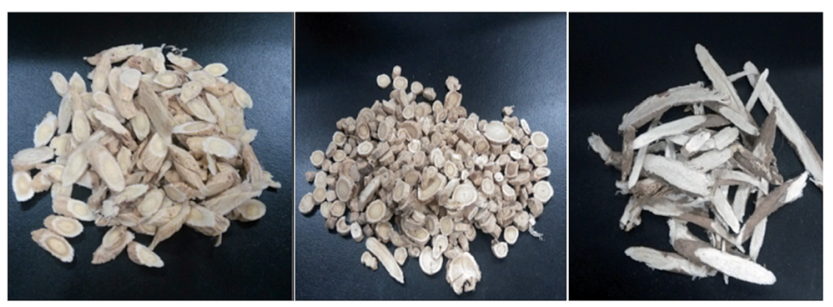

$\begin{array}{lll}\text { (1) from Gansu (2) from Shanxi } & \text { (3) from Sichuan }\end{array}$

Fig. 1 Appearance of Radix Astragali.
$12 \mathrm{~h}$, then fumigated for 2 days by the smoke generated from the combustion of a certain amount of sulfur in a homemade device. After that the fumigated Radix Astragali was dried in an air dry oven of $30^{\circ} \mathrm{C}$ for 2 days, milled with a household food disintegrator and sieved. The powder samples through 80-mesh sieve were collected and kept under seal.

During milling and sieving, it was found that more fibrous residue was not able to pass through 80-mesh sieve in Radix Astragali from Sichuan than from Gansu and Shanxi. The fibrous residue was cut with scissors and milled repeatedly until they can pass.

Five levels of sulfur fumigation were implemented for Radix Astragali of each habitat. The accurate sulfur dioxide residues in all the samples of Radix Astragali (three habitat, 5 levels of sulfur fumigation) were determined by acid-base titration method listed in Chinese Pharmacopoeia (2015) ${ }^{34}$ and the obtained values were treated as the reference values of sulfur dioxide residues.

The powder samples of certain appropriate levels of sulfur fumigation from a same habitat were weighed accurately, mixed according to a certain ratio to obtain 80 samples with different contents of $\mathrm{SO}_{2}$, so 240 spectral samples obtained for the three habitats.

\subsection{Apparatus}

S3000 fiber optic spectrometer (Race-Technology Co., Ltd, Hangzhou, China) equipped with a 3648-element linear silicon CCD array detector (Toshiba TCD 1305), a light source (Oceans Optics Inc., USA), a Y-type optical fiber probe and a home-made sample cell made from dark gray PVC was used to measure the UV-vis-SWNIR diffuse reflectance spectra. ${ }^{37}$

Sieves (Surwit Co., Ltd, Hangzhou, China) of 80, 100 and 120mesh sieves (0.200, 0.150 and $0.125 \mathrm{~mm}$ nominal diameters) were used to screen the milled samples.

\subsection{Procedures}

2.3.1 Spectral measurement. $0.150 \mathrm{~g}$ of each powder sample of Radix Astragali of each habitat was weighed and filled in the home-made sample cell and flattened with a flat round bar. Then the optical probe was placed vertically on the upper surface of the powder sample to measure UV-vis-SWNIR diffuse reflectance spectra in the range of $200-1100 \mathrm{~nm}$ under the following measuring conditions: exposure time of $250 \mathrm{~ms}$, integral sampling time of $1 \mathrm{~ms}$, average number of 15 and smoothing number of 15 , with a resolution of $0.33 \mathrm{~nm}$ and a spectralon as background reference. Each sample packed in the sample cell was measured twice before and after the sample was re-filled and the optical probe was reset, and their average was treated as a raw spectrum of the sample. In total, 240 spectra for the powder samples of Radix Astragali from three habitats were obtained.

2.3.2 Identification of habitats. Identification of habitats was carried out by applying principle component analysis (PCA) on the UV-vis-SWNIR diffuse reflectance spectra, using Unscrambler ver 9.7. 
2.3.3 Determination of $\mathrm{SO}_{2}$ residues. Determination of $\mathrm{SO}_{2}$ residues was implemented by applying partial least square regression (PLSR) modelling on the UV-vis-SWNIR diffuse reflectance spectra, using Unscrambler ver 9.7.

2.3.4 Evaluation of model performance. Root mean square error of prediction (RMSE), determination coefficient of prediction $\left(R^{2}\right)$ and relative predictive determinant of prediction (RPD) were used as model performance indicators. Their calculation can be found in the literature. ${ }^{38}$

According to Saeys et al. ${ }^{42}$ and Vohland et al., ${ }^{43} \mathrm{RPD}>3.0$ and $R^{2}>0.90$ are considered to be indicative of an excellent prediction; $2.5<\mathrm{RPD}<3.0$ and $0.81<R^{2}<0.90$ indicate good prediction; $2.0<\mathrm{RPD}<2.5$ and $0.66<R^{2}<0.81$ make approximate quantitative predictions possible; $1.5 \leq \mathrm{RPD}<2.0$ and $0.50 \leq R^{2}<0.66$ reveal a possibility to distinguish between high and low; RPD $<1.5$ or $R^{2}<0.50$ indicate that prediction is bad.

\section{Result and discussion}

\subsection{Spectra}

The original UV-vis SWNIR DRS showed that the absorbance in the range above $1090 \mathrm{~nm}$ and below $213 \mathrm{~nm}$ is negative. It might be due to instrument noise. Therefore, spectrum below $213 \mathrm{~nm}$ and above $1090 \mathrm{~nm}$ was cut off, leaving the spectral range of 213-1090 $\mathrm{nm}$ for analysis use, as shown in Fig. 2. It can be seen that the spectral waveforms were similar, but spectral intensity differs to some extend with habitats or $\mathrm{SO}_{2}$ residue levels, especially as for the samples from Sichuan in the ranges of 240$450 \mathrm{~nm}$ and 1000-1100 $\mathrm{nm}$.

\subsection{Identification of habitats}

Identification of habitats was carried out on the base of wavelength selection. The aim of wavelength selection is to select the informative wavelengths which are relevant to the property of interest, in other words, to remove the uninformative and/or interfering variables to construct a reliable and interpretable calibration model with good prediction accuracy.

The forward evolving window correlation coefficient method was used to select characteristic wavelength range for PCA. The

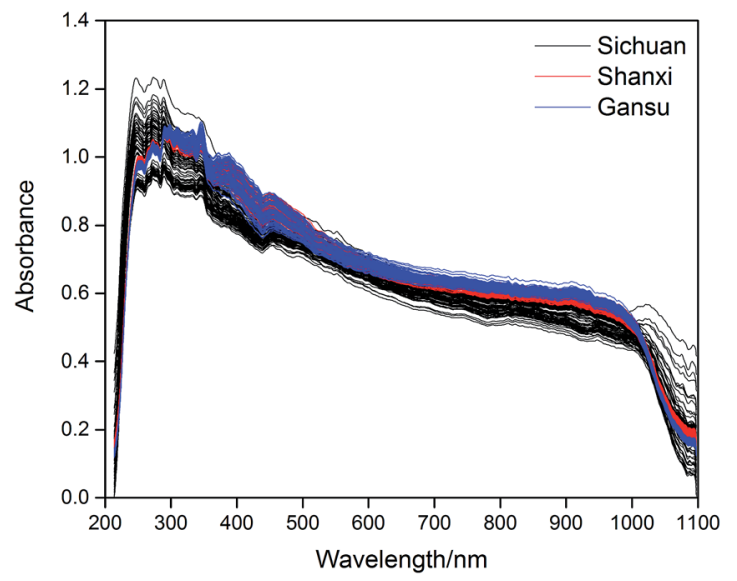

Fig. 2 Diffuse reflectance spectra of powder samples.

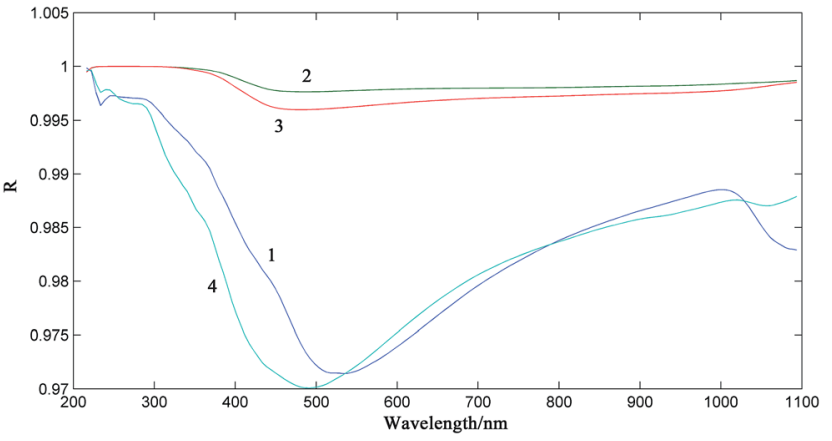

Fig. 3 Forward evolving window correlation coefficient method (1) Sichuan; (2) Shanxi; (3) Gansu; (4) average of inter-class.

intra-class correlation coefficient of every kind of habitat and the average of three inter-class correlation coefficients between every two kinds of habitat samples were calculated, the results are shown in Fig. 3.

The larger the intra-class correlation coefficient and the smaller the inter-class correlation coefficient, the more conducive the realization of sample classification and discrimination.

Fig. 3 shows that the correlation coefficients were all greater than 0.97 in the full spectral range, and in the range of 213$530 \mathrm{~nm}$ the average of inter-class correlation coefficients was smaller than the intra-class correlation coefficients. This provides the possibility to classify habitats. Therefore, besides the full spectral range of 213-1090 $\mathrm{nm}$, various ranges of 213$1090 \mathrm{~nm}, 213-590 \mathrm{~nm}, 213-510 \mathrm{~nm}, 320-510 \mathrm{~nm}, 213-350 \mathrm{~nm}$, 213-310 nm, 240-310 $\mathrm{nm}$ and 213-530 nm were tried as modelling wave bands in following PCA modelling.

In PCA modelling, the percentage of cumulative variance of the first $\mathrm{N}$ principle components $v s$. the total variance was calculated. Applying PCA in full spectral range of 213-1090 nm gave the first cumulative variance of $60 \%$, the second cumulative variance of $86 \%$ and the third cumulative variance of $94.5 \%$, while PCA in the range of 213-310 nm gave the best results, with the first cumulative variance of $72.5 \%$, the second cumulative variance of $95 \%$ and the third cumulative variance of $99 \%$. The three-dimensional projection using the range of $213-310 \mathrm{~nm}$ is shown in Fig. 4, which demonstrates the better effect of identification of habitats.

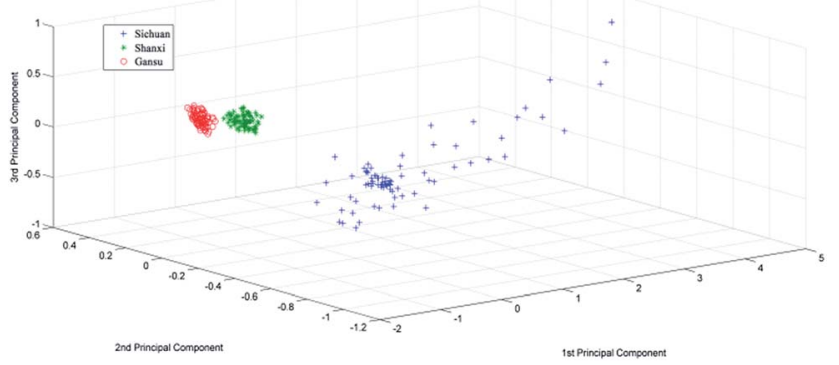

Fig. 4 Three-dimensional projection of the three habitats of Radix Astragali on the first three principle components of PCA in spectral range of $213-310 \mathrm{~nm}$. 
In Fig. 4, compared with Radix Astragali from Gansu and Shanxi, Radix Astragali from Sichuan dispersed in a larger scope, which may be due to the powder size of Radix Astragali from Sichuan. As has been mentioned above, more fibrous residue in Sichuan Radix Astragali powder was found than in Gansu Radix Astragali, Shanxi Radix Astragali. Therefore, it was considered that there were more fibrous or long-shaped particles in Sichuan Radix Astragali powder samples. This is disadvantageous to obtain a smooth sample surface when filled in the sample cell. Rough surface of a packed sample may cause larger fluctuations of the measured spectra. But in this case, this still permit a clear discriminant of the three habitats of Radix Astragali. This implies that the difference of natures or compositions between the three habitats of Radix Astragali is larger than the difference caused by smoothness of sample surface.

The results show that UV-vis spectrum plays an important role in identification of origin of Radix Astragali; by using UVvis-SWIR DRS combined with PCA, habitat identification of Radix Astragali is feasible, whether it is sulfur-fumigated, or not sulfur-fumigated.

\subsection{Determination of $\mathrm{SO}_{2}$ residues}

The total 240 spectra of Radix Astragali from 3 habits were divided into a calibration set of 180 samples and a test set of 60 samples by K/S algorithm.

3.3.1 Wavelength selection. Wavelength selection for PLSR model was carried out by correlation coefficient method and iPLS method, respectively.

Fig. 5 is the plot of the correlation coefficient between $\mathrm{SO}_{2}$ residue and absorbance $v s$. wavelength. The bands with a $R^{2}$ greater than 0.2421 , that is, the mean value of absolute values of all $R^{2}$ in the full spectrum, were used to build PLSR model for prediction of $\mathrm{SO}_{2}$ residue. But the results were not satisfactory: the square correlation coefficient $R^{2}$ between predicted values of $\mathrm{SO}_{2}$ residue and their reference values for the calibration set and prediction set was 0.7805 and 0.7406 , respectively.

Then, wavelength selection for PLSR model was carried out by iPLS method. Fig. 6 and 7 is the plot of RMSECV vs. wavelength when the full wavelength range was divided into 2 intervals (2-iPLS) and 3 intervals (3-iPLS), respectively.

Comparing Fig. 6 and 7, it can be known that the RMSECV with the band (213-691 nm) obtained by 2-iPLS and the

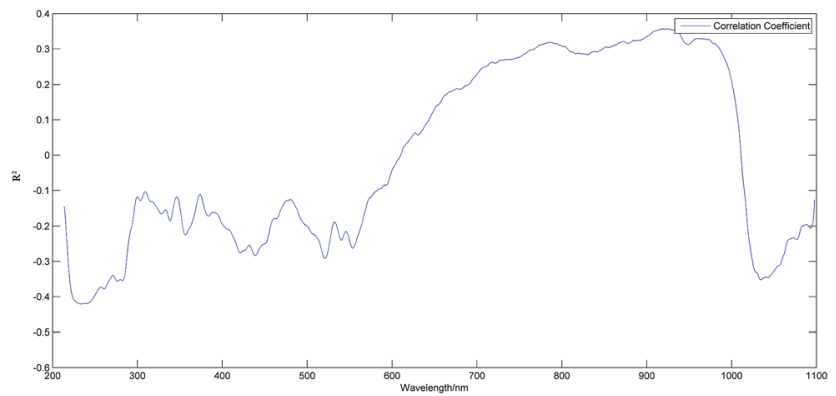

Fig. 5 Correlation coefficient method for wavelength selection.

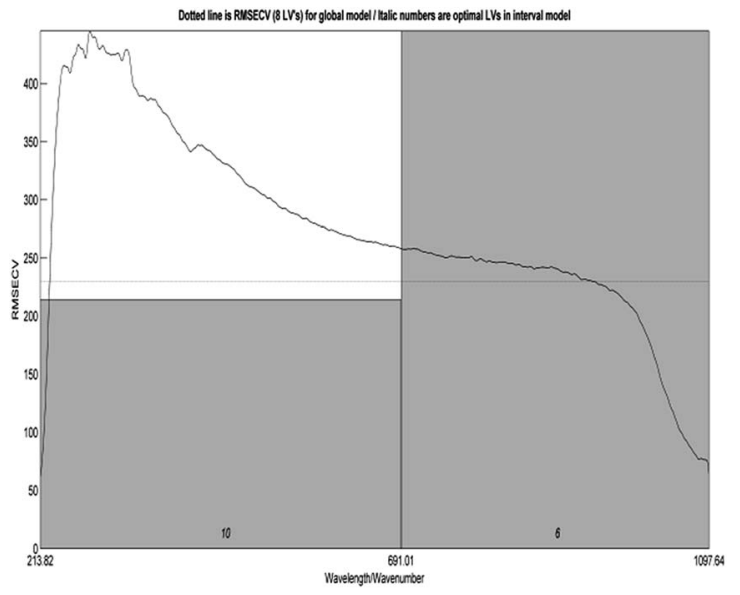

Fig. 6 Modelling effect by 2-iPLS method.

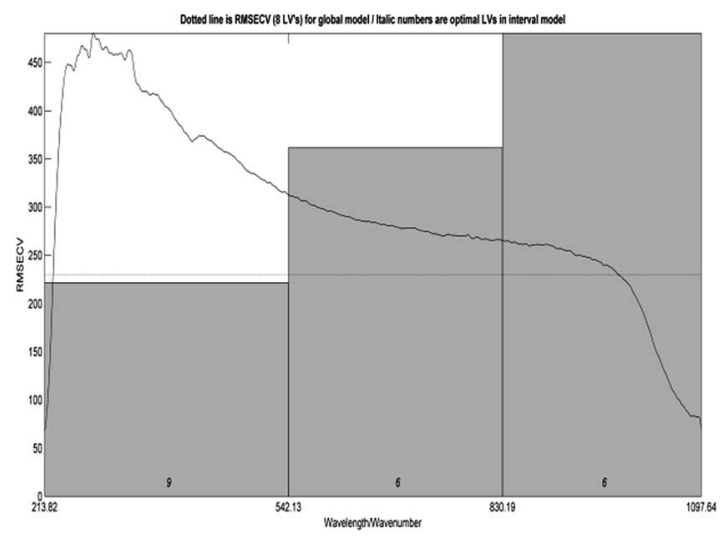

Fig. 7 Modelling effect by 3-iPLS method.

RMSECV with the band (213-542 $\mathrm{nm}$ ) by 3-band iPLS were both lower than that (horizontal line) with the whole band, but the former was lower. So the band of 213-691 nm selected by 2-iPLS method was used in further study, and the modelling effects were listed at the fourth line in Table 1, better than those using the wavelength selected by correlation coefficient method (Fig. 5).

3.3.2 Spectral data pre-treatment. Ultraviolet-visible spectra contain not only sample information but also some irrelevant information and noise, such as instrument noise, sample background and stray light. Therefore, it's significant to select spectral pre-treatment methods to build robust quantitative model.

In this study, spectral data pre-treatment was performed by the 18 pre-treatment methods shown in Table 1, using the optimum modelling waveband (213-691 nm) selected in Section 3.3.1. It is found that modelling with SG smoothing pretreatment has the best effect.

3.3.3 Outliers exclusion. Generally, as for quantitative analysis based on spectra, there may be some outliers consisting of spectral outliers and chemical value outliers. F-Test and PCA coupled with Mahalanobis Distance (MD) is applied on 
Table 1 Modelling and verification of determination of residual $\mathrm{SO}_{2}$ in Radix Astragali by PLS ${ }^{a}$

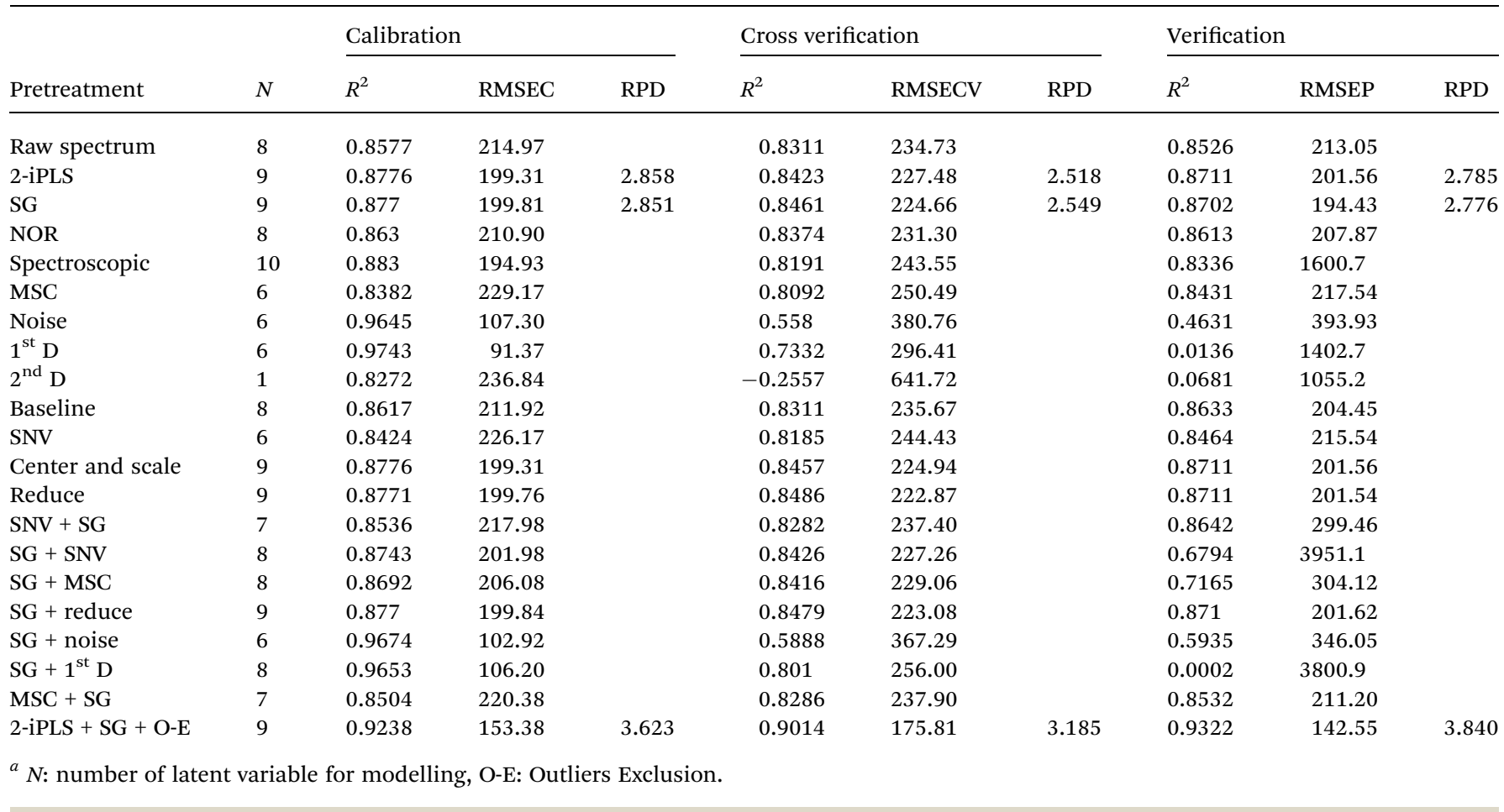

identifying chemical value outliers and spectral outliers, respectively. In the calculation process of PCA and MD, the number of PCs is selected according to the cumulative contribution of PCs set as $95 \%$, and the threshold $\left(D_{\mathrm{t}}\right)$ of MD is decided by setting the weight coefficient $t$ as $2 .{ }^{44}$

In this study, the outlier removal was carried out on the basis of SG smoothing pre-treatment having the best modelling effect. By computation, the value of $D_{\mathrm{t}}$ was 2.7789 . The chemical value outliers were determined by the threshold value $\left(F_{\mathrm{a}}=\right.$ 4.0099) computed by $F$ distribution with 1 degree of freedom in the numerator and 239 degrees of freedom in the denominator and $95 \%$ of confidence level. As a result, there were 21 outliers as shown in Fig. 8. The results of modelling before and after outlier exclusion were showed in Table 1.

3.3.4 Modelling effects. After waveband selection, SG smoothing and excluding outliers, modelling results were improved with a $R^{2}$ of 0.9238 for calibration set, a $R^{2}$ of 0.9104 for cross validation set and a $R^{2}$ of 0.9322 for validation set as shown in Table 1. Additionally, RPD by calculation was 3.8405 . According to Saeys et al. and Vohland et al., $R^{2}>0.90$ and RPD > 3.0 at same time are considered to be indicative of an excellent prediction. The results demonstrate that an excellent prediction has been achieved. The plots of predicted values of $\mathrm{SO}_{2}$ residue $v s$. their reference values for the calibration set and prediction set are gave in Fig. 9 and 10.

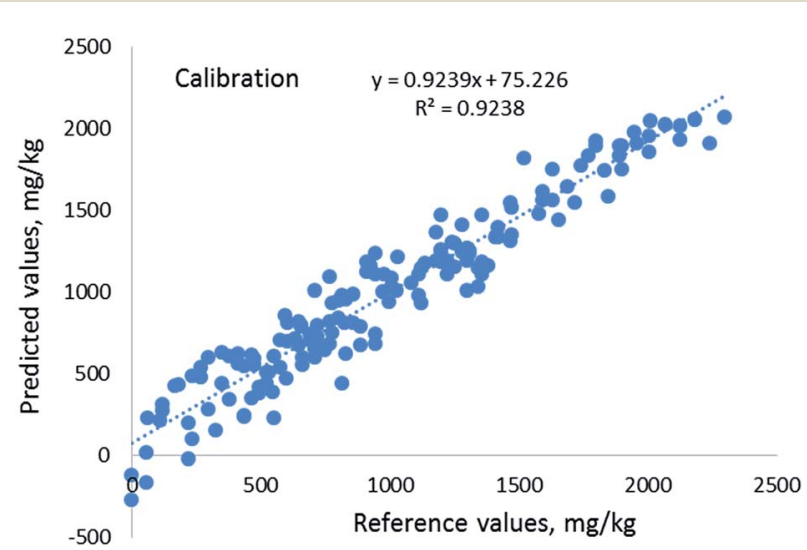

Fig. 9 Plot of predicted value vs. reference value of calibration set by PLS.

Fig. 8 Outliers exclusion. 


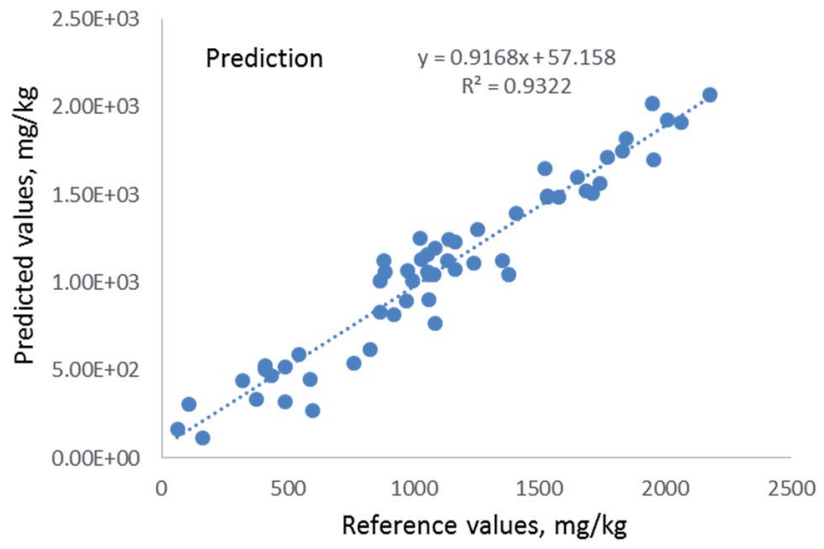

Fig. 10 Plot of predicted value vs. reference value of prediction set by PLS.

The limit of detection (LD) was calculated to estimate the lowest content of residual $\mathrm{SO}_{2}$ that can be measured. The limit of detection was determined from multiple measurements $(n=$ 9) of the spectral response of samples containing $60 \mathrm{mg}$ residual $\mathrm{SO}_{2}$ per kg of Radix Astragali from Gansu and Shanxi, $267 \mathrm{mg}$ residual $\mathrm{SO}_{2}$ per $\mathrm{kg}$ of Radix Astragali from Sichuan. The LDs were calculated by multiplying the standard deviation by three and then dividing by the slope of the calibration curve, as shown by eqn (1):

$$
\mathrm{LD}=3 \times \text { S.D./slope }
$$

The LD of residual $\mathrm{SO}_{2}$ was found to be $360 \mathrm{mg} \mathrm{kg}^{-1}$ for Radix Astragali from Gansu and Shanxi, $550 \mathrm{mg} \mathrm{kg}^{-1}$ for Radix Astragali from Sichuan. Although they are not as low as $150 \mathrm{mg}$ $\mathrm{kg}^{-1}$, but less than or near $400 \mathrm{mg} \mathrm{kg}{ }^{-1}$, the limit of sulfur dioxide residues in ten traditional Chinese medicines allowed by China. Compared with the qualitative analysis of sulfur dioxide residues in the report ${ }^{35}$ which used FTIR, the sensitivity of our method is greatly improved. It is believed that in future with the improvement of optical fiber spectrometer hardware system, especially the improvement of the stabilities of light source and spectrometer, the precision of the proposed method will be improved, consequently, the detection limit will be reduced.

\section{Conclusions}

The application of UV-vis and short wave near-infrared diffuse reflectance spectrum coupled with chemometrics on the identification of habitat and determination of sulfur dioxide residue of sulfur fumigated Chinese herb medicine was investigated first. UV-vis spectrum plays an important role in both the identification of habitat of CHM and determination of $\mathrm{SO}_{2}$ residue. By using UV-vis-SWNIR DRS combined with PCA, habitat of Radix Astragali is identified definitely, whether it is sulfur-fumigated, or not sulfur-fumigated; through wavelength band selection, SG smoothing and excluding outliers, modelling results were improved with a $R^{2}$ of $0.9238,0.9104$ and 0.9322 for calibration set, cross validation set and validation set, respectively.
The results imply that a convenient, rapid method for the identification of habitat of sulfur fumigated Chinese herbal medicines and determination of sulfur dioxide residue by UVvis and short wave near-infrared diffuse reflectance spectrum is feasible.

\section{Acknowledgements}

This work was supported by the Science and technology special fund of Sichuan Provincial Administration of Traditional Chinese Medicine, China (No. 2016Q058) and the Scientific Research Foundation of the Education Department of Sichuan Province, China (No. 16ZB0122).

\section{References}

$1 \mathrm{~J} . \mathrm{Xu}$, Authenticity identification of Chinese rhubarb, Tianjin Pharmacy, 2001, 6, 51-52.

2 J. R. Wang, Z. Y. Zhang, Z. W. Zhang, Y. H. Xiang and P. d. B. Harrington, THz-TDS combined with a fuzzy rulebuilding expert system applied to the identification of official rhubarb samples, Anal. Methods, 2014, 6, 7695.

3 Y. L. Zhen, C. X. Zhang and X. G. Li, TLC scanning method for identification of Panax quinquefolius Linn. and $\mathrm{P}$. ginseng Meyer, Chin. Tradit. Pat. Med., 1989, 7, 35-36.

4 S. L. Wang, TLC identification of radix ginseng, radix notoginseng and radix astragali, Drug Stand. China, 2003, 2, 48-50.

5 X. Su, X. K. Li, T. Wu, T. Zhang and Z. T. Wang, Fingerprint analysis of Glehniae Radix by TLC, J. Chin. Med. Mater., 2012, 35, 210-213.

6 Y. L. Feng and B. Y. Yu, Identification of Rhei and some preparations contained Rhei by HPLC, Drug Stand. China, 2009, 10, 296-298.

7 F. Q. Zha, L. F. Hu, J. W. Wu and Y. X. He, Study on identification of genuine and sham flos chrysanthemi indici by HPLC, J. Anhui Agric. Sci., 2011, 39, 5765-5766.

8 L. R. Duan, X. K. Zhang, W. Cao, Y. H. Xie and S. W. Wang, Study on the HPLC fingerprint of toad skin from different regions, J. Chin. Med. Mater., 2012, 35, 182-187.

9 L. Yang, Z. J. Su, X. Zeng, X. Li and Z. F. Wu, Quality assessment of Fructus xanthii based on fingerprinting using high-performance liquid chromatography, J. AOAC Int., 2012, 95, 1053-1058.

10 Q. Li, Y. Q. Liu, L. F. Han, J. Z. Liu, W. Y. Liu, F. Feng, J. Zhang and N. Xie, Chemical constituents and quality control of two Dracocephalum species based on highperformance liquid chromatographic fingerprints coupled with tandem mass spectrometry and chemometrics, J. Sep. Sci., 2016, 39, 4071-4085.

11 D. Wang, Q. Y. Zhang, X. X. Yang, Y. J. Jiang, M. Y. Shang, Z. W. Zhang, S. Q. Cai, Y. Y. Zhao and X. Wang, Study on HPLC-fingerprint-based identification of Dao-di herb and non Dao-di herb of Scutellariae Radix, China J. Chin. Mater. Med., 2013, 12, 1951-1960.

$12 \mathrm{H}$. Q. Chen, Study on infrared fingerprint of Salvia miltiorrhiza, China J. Chin. Mater. Med., 2006, 31, 1285-1286. 
13 K. Y.-L. Yap, S. Y. Chan and C. S. Lim, Authentication of traditional Chinese medicine using infrared spectroscopy: distinguishing between ginseng and its morphological fakes, J. Biomed. Sci., 2007, 14, 265-273.

$14 \mathrm{~J}$. Mao and J. Xu, Discrimination of herbal medicines by molecular spectroscopy (including near infrared diffuse reflection spectroscopy, Raman spectroscopy and infrared spectroscopy) and chemical pattern recognition, Spectrochim. Acta, Part A, 2006, 65, 497-500.

15 H. R. Zhao, S. M. Wen, X. Y. Wang, S. M. Chu and X. J. Huo, Study of Flos Lonicerae in local herbs and other areas by FTIR spectrum, Spectrosc. Spectral Anal., 2005, 25, 705-707.

16 Y. A. Woo, H. J. Kim, J. H. Cho and H. Chung, Discrimination of herbal medicines according to geographical origin with near infrared reflectance spectroscopy and pattern recognition techniques, J. Pharm. Biomed. Anal., 1999, 21, 407-412.

17 B. K. Huang, B. Zhu, H. C. Zheng, L. P. Qin and Q. Y. Zhang, Identification of Fructus Broussonetiae from different habitats and its confused species with clustering analysis by near-infrared diffuse reflectance spectrometry, J. Chin. Med. Mater., 2002, 25, 874-875.

18 L. Y. Sun, T. M. Yang and Y. Y. Wang, Identification of Cortex Phellodendri Chinensis by near infrared spectroscopy fingerprint, Comput. Appl. Chem., 2008, 25, 329-332.

19 B. X. Li, Y. H. Wei, L. L. Xi, H. G. Duan and X. A. Wu, Qualitative research on Radix Anglicae Sinensis from different origins in different harvest time by near infrared spectrum and chemometrics, Chin. J. Spectrosc. Lab., 2011, 28, 2128-2134.

20 H. Li, S. Q. Du, L. Xie, L. Chen, Y. Peng, Y. M. Zhu, T. Wu, H. Li, P. Dong and J. T. Wang, Identifying Radix Curcumae by using terahertz spectroscopy, Optik, 2012, 123, 1129-1132.

21 Q. K. Chen, Y. M. Zhu, H. Jia, S. Q. Du, G. M. Liu and S. L. Zhuang, Identifying Radix Curcumae based on partial least squares, Optik, 2013, 124, 3091-3094.

22 Y. J. Chen, Y. Y. Liu, G. Z. Zhao, W. N. Wang and F. L. Li, Chinese traditional medicine recognition by support vector machine(SVM) Terahertz spectrum, Spectrosc. Spectral Anal., 2009, 29, 2346-2350.

23 Y. P. Yang, S. Sree Harsh, A. J. Shutler and D. R. Grischkowsky, Identification of Genistein and Biochanin A by $\mathrm{THz}$ (far-infrared) vibrational spectra, $J$. Pharm. Biomed. Anal., 2012, 62, 177-181.

24 J. Jiang, P. Li, H. B. Zhou, Y. Y. Qian, L. Sun, R. Wan, S. L. Li and X. B. Jia, Study condition and the quality control study route of the sulfur fumigation Chinese medicine, China J. Tradit. Chin. Med. Pharm., 2014, 29, 1764-1767.

25 C. Q. Mao, L. Ji, T. L. Lu, X. Shan, L. Li, H. Z. Liu, Z. W. Ning, Y. Song and J. J. Zhu, Research development of harmful substances and its harm of traditional Chinese medicine after sulfur fumigation, China J. Chin. Mater. Med., 2014, 39, 2801-2806.

26 L. Ji, D. X. Li, Y. Peng and L. Tong, Advances in Study of Sulfur Dioxide Residue in Traditional Chinese Medicine and its Prepared Slices, Mod. Chin. Med., 2015, 17, 185-190.
27 S. L. Li, J. Z. Song, F. F. K. Choi, C. F. Qiao, Y. Zhou, Q. B. Han and $\mathrm{H}$. X. Xu, Chemical profiling of Radix Paeoniae evaluated by ultra performance liquid chromatography/ photo diode array/quadru pole time of flight mass spectrometry, J. Pharm. Biomed. Anal., 2009, 49, 253-266.

28 Z. X. Xing, Y. B. Cui and W. Liu, Determination of the content of Chlorogenic acid in sulfur fumigation and drying honeysuckle of different areas by HPLC, China Med. Her., 2013, 6, 98-99.

29 X. H. Wang, P. S. Xie, W. K. Lam Chris, C. W. K. Lama, Y. Z. Yan and Q. X. Yu, Study of the destructive effect to inherent quality of Angelicae dahur icae radix(Baizhi) by sulfur fumigated process using chromatographic finger printing analysis, J. Pharm. Biomed. Anal., 2009, 49, 12211225.

30 Y. Peng, X. L. Li, L. Yin, Z. Wei and Y. P. Liu, Study on the research situation of sulfur fumigated processing and detection method of sulfur dioxide residues in traditional Chinese medicine, Pharmacy and Clinics of Chinese Materia Medica, 2012, 5, 5-8.

31 L. Xiao, T. Jiang, J. Nie and Y. Cheng, Impact of sulfur fumigation on quality of Scutellariae Radix, China J. Chin. Mater. Med., 2016, 41, 2216-2220.

32 Y. Duan, K. M. Qin, N. S. Zou, Y. J. Lou, H. Cai and B. C. Cai, Sulfur-fumigation, maintenance method of Chinese herbal medicine-discard or inheritance, China J. Chin. Mater. Med., 2013, 38, 3395-3399.

33 X. Liu, X. Q. Ma, H. Cai, J. J. Liu, J. S. Li and B. C. Cai, Determination of metallic and trace elements in Lonicerae japonicae Flos before and after sulfur-fumigated process by ICP-AES, Chin. Tradit. Pat. Med., 2012, 34, 293-296.

34 Chinese Pharmacopoeia, 2015 version.

35 K. Pei, H. Cai, X. Liu, Y. J. Lou, X. Q. Song, F. X. Qiao and B. C. Cai, Identification of sun-dried and sulfur-fumigated Chuanxiong Rhizoma by FTIR, J. Chin. Med. Mater., 2015, 38, 1393-1399.

36 Y. J. Lou, H. Cai, X. Liu, K. Pei, X. Q. Ma, S. L. Li and B. C. Cai, Quick identification of sun-dried and sulfur-fumigated Angelicae Sinensis Radix by Fourier transform infrared spectroscopy, China J. Chin. Mater. Med., 2012, 37, 11271132.

37 Y. Y. Feng, X. L. Li, K. L. Xu, H. Y. Zou, H. Li and B. Liang, Qualitative and simultaneous quantitative analysis of cimetidine polymorphs by ultraviolet-visible and shortwave near-infrared diffuse reflectance spectroscopy and multivariate calibration models, J. Pharm. Biomed. Anal., 2015, 104, 112-121.

38 X. L. Li, K. L. Xu, H. Li, S. Yao, Y. F. Li and B. Liang, Qualitative analysis of chiral alanine by UV-visible shortwave near infrared diffuse reflectance spectroscopy combined with chemometrics, RSC Adv., 2016, 6, 8395-8405.

39 J. Tang, X. Li, Y. Feng and B. Liang, Simultaneous determination of amiloride and hydrochlorothiazide in a compound tablet by diffuse reflectance spectroscopy and chemometrics, J. Appl. Spectrosc., 2016, 83, 669-675.

40 B. Liang, X. L. Li, X. L. Jing, T. Li and S. Yao, Qualitative and quantitative analysis of chiral alanine by UV-visible- 
shortwave near infrared diffuse reflectance spectroscopy combined with chemometrics, J. Sichuan Univ., Eng. Sci. Ed., 2016, 3, 190-195.

41 T. Li, R. F. Tu, J. Zhu and B. Liang, Rapid and simultaneous determination of soil properties by UV-vis-SWIR diffuse reflectance spectroscopy, Chemical Research and Application, 2016, 28, 987-993.

42 W. Saeys, A. M. Mouazen and H. Ramon, Potential for onsite and online analysis of pig manure using visible and near infrared reflectance spectroscopy, Biosyst. Eng., 2005, 91, 393-402.

43 M. Vohland, M. Ludwig, S. Thiele-Bruhn and B. Ludwig, Determination of soil properties with visible to near- and mid-infrared spectroscopy: Effects of spectral variable selection, Geoderma, 2014, 223-225, 88-96.

44 W. L. Zhang, N. Li, Y. Y. Feng, S. J. Su, T. Li and B. Liang, A unique quantitative method of acid value of edible oils and studying the impact of heating on edible oils by UV-vis spectrometry, Food Chem., 2015, 185, 326-332. 